\title{
Determination and Combination of Quantitative Weight Value from Multiple Preference Information
}

\author{
Ji Hyun Yoo', Byong Gul Lee ${ }^{1}$, and Hyuk Soo $\operatorname{Han}^{2}$ \\ ${ }^{1}$ Department of Computer Science and Engineering, \\ Seoul Women's University, 126 Nowon-Gu, GongReung 2 dong, Seoul, Korea \\ $\{$ jhyu, byongl\}@swu.ac.kr \\ ${ }^{2}$ Department of Software Science, Sang Myung University, \\ Hongji-dong 7, Seoul, Korea \\ hshan@smu.ac.kr
}

\begin{abstract}
Recently, the concern of software quality increases rapidly. Although there have been many efforts to establish standards for software quality, such as ISO/IEC 9126, they provide only a framework for quality characteristics and evaluation process. They do not provide practical guidance for deriving reasonable weight value criteria for quality evaluation. 1 This paper presents a method to draw the quantitative weight values from evaluator's subjective data in software evaluation in compliance with ISO/IEC 9126 standard. To eliminate evaluators' subjectiveness and uncertainty, the Dempster-Shafer (D-S) theory is improvised and utilized. The D-S theory is improved with merge rule to reduce the bias of weight value when they are merged with other evaluator's weight value. The proposed merge rule has been tested for it's effectiveness with actual evaluation data.
\end{abstract}

\section{Introduction}

Software quality is defined as a specification of functions of which software performs. High quality software means that it not only satisfies its specification, but also achieves its quality characteristics, such as functionality, reliability, usability, efficiency, maintainability, portability. To evaluate software quality based on these quality characteristics, various standards and techniques can be applied. ISO/IEC 9126 [1], for example, provides a standard for establishing software quality characteristics and metrics. ISO/IEC 14598 [2] provides methods and procedures for performing of quality authentication. These standards, however, does not specify specific evaluation technique such as the calculation of weight value for evaluation items. Consequently, software quality evaluation tends to depend on assessor's subjective judgment and knowledge. That is, the interpretation of evaluation criteria, semantic relation between evaluation items, and measurement depends on evaluators' viewpoint [3], [4], [5]. Existing studies also overlook the problem of producing incorrect result in combining different weight values from multiple assessors [6], [7], [8].

\footnotetext{
1 This research was supported by University IT Research Center Project
} 
This paper describes a quantitative method for calculating the weight value of evaluation items by combining different evaluation values which is retrieved from multiple assessors' subjective opinion. Specifically, this paper discusses how to improve the way of aggregating and combining multiple assessors' opinions considering the relations between the evaluation items.

\section{Related Works}

\subsection{Inference Using Bayes' Theory}

Bayes' theory [11], [12], [13] is a statistical inference technique to estimate the probability of certain event occurring in which different hypotheses are given for each evidence. Assuming that there exists mutually exclusive hypothesis $H_{k}(k=1,2, \ldots, n)$ for a certain evidence E, then Bayes' theory is described as following (1).

$$
P\left(H_{n} \mid E\right)=\frac{\left(P\left(E \mid H_{n}\right) \cdot P\left(H_{n}\right)\right)}{\sum_{k=1}^{n} P\left(E \mid H_{k}\right) \cdot P\left(H_{k}\right)}
$$

From the above expression, $\mathrm{H}_{\mathrm{n}}$ and $\mathrm{E}$ are related in cause and effect relation. Then, $\mathrm{P}\left(\mathrm{H}_{\mathrm{n}}\right)$ is called as a priori probability and $\mathrm{P}\left(\mathrm{H}_{\mathrm{n}} \mid \mathrm{E}\right)$ as a posteriori probability. Bayes' theory, however, has a few problems in its application. First, if there are n numbers of evidence and $m$ numbers of hypothesis, it is necessary to know in prior $n * m$ numbers of probability. This means that a large number of evidence and hypothesis requires a great number of priori probability calculation. In reality, it is not possible to provide all priori probability values in advance. Second, the probability value in Bayes' theory is assumed to be mutually exclusive. That is, if the probability of a certain event occurring be 0.5 , then the probability for the event not occurring is $1-0.5=0.5$. However, in real situation, the probability for an event not occurring isn't always 0.5 since it is not known whether a certain event occurrs or not. Finally, there is no rule for successively accruing the probability value in Bayes' theory. That is, if a new hypothesis is introduced, the Bayes theory has to compute the probability from the scratch again.

\subsection{Quantitative Translation from Qualitative Relationship}

The technique of deriving quantitative value from qualitative relationship information can be achieved by using Dempster Shafer (DS) theory [9], [10], [14], [17]. It uses the qualitative preference relations such as "A is more important than $\mathrm{B}$ " or " $\mathrm{A}$ is similar to C."

DS theory compensates the drawbacks of Bayes' theory by defining the belief of hypothesis on $\mathrm{H}$ to an interval between $\operatorname{Bel}(\mathrm{H})$ and $\mathrm{Pl}(\mathrm{H})$. $\mathrm{Bel}(\mathrm{H})$ is a belief value of a given evidence and $\mathrm{Pl}(\mathrm{H})$ is a plausibility value based on evidence. DS theory also provides a combination rule on merging two random variables that are independent each other (2). A function $\mathrm{m}: 2^{\mathrm{s}} \rightarrow[0,1]$ ( $\mathrm{S}$ is a frame of discernment) is called a basic probability assignment [11], [12], [13], [15], [16]. 


$$
m 3=\frac{\sum_{s 1 \cap s 2=s 3} m 1(s 1) \cdot m 2(s 2)}{1-\sum_{s 1 \cap s 2=\phi} m 1(s 1) \cdot m 2(s 2)}
$$

This combination rule, however, may generate an empty set after intersection of two discernments [7], [8]. This can degrade the correctness of combination results. In addition, none of intending hypothesis can have total value, 1, after normalization process. This means that the lower supporting discernments can produce the higher supporting value after the combination as shown in table 1.

Table 1. Result of normal combination rule in DS theory

\begin{tabular}{l|l|c}
\hline $\mathrm{m} 2$ & $\mathrm{~m} 1(\{\mathrm{a}\})=0.1$ & $\mathrm{~m} 1(\{\mathrm{~b}\})=0.9$ \\
\hline $\mathrm{m} 2(\{\mathrm{a}\})=0.1$ & $\mathrm{~m} 3(\{\mathrm{a}\})=0.01$ & $\mathrm{~m} 3(\varnothing)=0.09$ \\
\hline $\mathrm{m} 2(\{\mathrm{c}\})=0.9$ & $\mathrm{~m} 3(\varnothing)=0.09$ & $\mathrm{~m} 3(\varnothing)=0.81$ \\
\hline Normalization & $\mathrm{m} 3(\{\mathrm{a}\})=0.01 / 0.01=1$ \\
\hline
\end{tabular}

\section{Improved Combination Rule in DS}

As described earlier, the normal combination rule in DS theory can generate empty sets when different discernments are combined. The improved combination rule can reflect assessor's different opinions by redistributing the empty set value to the discernment. Table 2 shows the result of combining the exclusive discernments using the improved combination rule. Let the basic probability assignment (bpa) assigned by assessor $A$ be $\mathrm{m} 1$ and bpa by assessor $\mathrm{B}$ be $\mathrm{m} 2$. From the table 2 , since $\mathrm{m} 1(\{\mathrm{~b}\})=0.9$, $\mathrm{m} 2(\{\mathrm{c}\})=0.9$, the result of combination of $\mathrm{m} 1$ and $\mathrm{m} 2$ is $\mathrm{m} 3(\{\varnothing\})=0.81$. This means that assessor A assigns the wider opinion to discernment $\mathrm{b}$ and assessor $\mathrm{B}$ assigns the wider opinion to discernment c. In common, it assigns the narrower opinion to discernment a since the value of $\mathrm{m} 3(\{\varnothing\})$ is redistributed to the discernment $\mathrm{b}$ and $\mathrm{c}$. In the same way, $m 3(\{b\})=0.405$ and $m 3(\{c\})=0.405$. The final result after the combination shows in table 2 .

\section{Weight Value Determination in Software Quality Evaluation}

This chapter describes an example in calculating the weight value of software quality characteristics such as reliability defined in ISO/IEC 9126. For calculating the weight value, the first step is to determine the focal elements from assessors' qualitative preference evaluation. After determining the focal elements, the basic probability assignment (bpa) value is computed [9], [10], [14], [17]. If there is more than one assessor, then apply the improved combination rule in combining the bpas. Finally, compute a weight value using the belief function (Bel) and the probability function (Pl). 
Table 2. Result of combination about exclusive discernment using improved combination rule in DS theory

\begin{tabular}{|c|c|c|}
\hline $\mathrm{m} 2 \mathrm{~m}^{\mathrm{m}}$ & $\mathrm{m} 1(\{\mathrm{a}\})=0.1$ & $\operatorname{m1}(\{b\})=0.9$ \\
\hline \multirow{3}{*}{$\mathrm{m} 2(\{\mathrm{a}\})=0.1$} & $\mathrm{~m} 3(\{\mathrm{a}\})=0.01$ & $\mathrm{~m} 3(\varnothing)=0.09$ \\
\hline & & $\mathrm{m} 3(\{\mathbf{a}\})=0.009$ \\
\hline & & $\mathrm{m3}(\{b\})=0.081$ \\
\hline \multirow{3}{*}{$\mathrm{m} 2(\{\mathrm{c}\})=0.9$} & $\mathrm{~m} 3(\varnothing)=0.09$ & $\mathrm{~m} 3(\varnothing)=0.81$ \\
\hline & $\mathrm{m} 3(\{\mathrm{a}\})=0.009$ & $\mathrm{~m} 3(\{b\})=0.405$ \\
\hline & $\mathrm{m} 3(\{c\})=0.081$ & $\mathrm{~m} 3(\{\mathrm{c}\})=0.405$ \\
\hline \multirow{4}{*}{$\begin{array}{l}\text { Result of improved } \\
\text { combination rule }\end{array}$} & \multicolumn{2}{|c|}{$\mathrm{m} 3(\{\mathrm{a}\})=0.01+0.009+0.009=\mathbf{0 . 0 2 8}$} \\
\hline & \multicolumn{2}{|c|}{$\mathrm{m} 3(\{\mathrm{~b}\})=0.081+0.405=\mathbf{0 . 4 8 6}$} \\
\hline & \multicolumn{2}{|c|}{$\mathrm{m} 3(\{\mathrm{c}\})=0.801+0.405=\mathbf{0 . 4 8 6}$} \\
\hline & \multicolumn{2}{|c|}{$\mathrm{m} 3(\{\mathrm{a}\})+\mathrm{m} 3(\{\mathrm{~b}\})+\mathrm{m} 3(\{\mathrm{c}\})=0.028+0.486+0.486=1$} \\
\hline
\end{tabular}

\section{(1) Determining the Focal Elements and bpa}

Given assessor's qualitative preference relationship, the focal element can be determined using the following definition (3) and (4).

$\mathrm{A} \cdot>\mathrm{B} \leftrightarrow \mathrm{A}$ is higher weight value than $\mathrm{B}$.

A S $\mathrm{B} \leftrightarrow(\mathrm{j}(\mathrm{A}>\mathrm{B}) \& \mathrm{j}(\mathrm{B}>\mathrm{A})) \leftrightarrow \mathrm{A}$ is similar weight value to $\mathrm{B}$

Let $\mathrm{T}=\{\mathrm{a}, \mathrm{b}, \mathrm{c}, \mathrm{d}\}$ be the discernment and assume that an assessor has defined preference relationship as in (5).

$\{a\}>\{d\},\{b, c\}>\{b\},\{c, d\}<\{d\},\{a, b, c\} S\{b, c\},\{b\} S\{d\},\{b\}>0,\{d\}>0$

To determine the focal elements, it is necessary to eliminate illogical elements by using the elimination theorem [9], [10], [14], [17]. From the theorem, since \{c, $d\}<\{d\}$ and $\{a, b, c\} S\{b, c\},\{c, d\}$ and $\{a, b, c\}$ are not focal elements. In this way, the complete focal elements can be achieved: $\{a\},\{b\},\{b, c\},\{c\},\{d\}$.

After achieving the focal elements, the bpa of $\mathrm{ml}$ can be obtained using the equality and perceptron algorithm as following.

$$
\text { bpa of } m 1=\left[\begin{array}{c}
m 1(\{a\}) \\
m 1(\{b\}) \\
m 1(\{b, c\}) \\
m 1(\{c\}) \\
m 1(\{d\})
\end{array}\right]=\left[\begin{array}{l}
2 \\
1 \\
2 \\
2 \\
1
\end{array}\right]=\left[\begin{array}{c}
0.25 \\
0.125 \\
0.25 \\
0.25 \\
0.125
\end{array}\right]
$$




\section{(2) Application of the Improved Combination Rule}

In case there is more than one assessor, then the improved combination rule is applied. Let's assume that other assessor's qualitative preference relationship is given as (6).

$$
\{a\} S\{b\},\{b, c\}>\{a\},\{b, c\}<\{b\},\{c\} S\{d\},\{a\}>0,\{d\}>0
$$

The bpa and $\mathrm{m} 2$ can be obtained by repeating step (1).

$$
\text { bpa of } m 2=\left[\begin{array}{c}
m 2(\{a\}) \\
m 2(\{b\}) \\
m 2(\{b, c\}) \\
m 2(\{c\}) \\
m 2(\{d\})
\end{array}\right]=\left[\begin{array}{c}
1 \\
1 \\
2 \\
2 \\
2
\end{array}\right]=\left[\begin{array}{c}
0.125 \\
0.125 \\
0.25 \\
0.25 \\
0.25
\end{array}\right]
$$

Applying the improved combination rule to the bpa of $\mathrm{m} 1$ and $\mathrm{m} 2$, the results of the bpa of $\mathrm{m} 3$ is shown blow. To evaluate other assessor's value, repeat step (1) and (2).

$$
\text { bpa of } m 3=\left[\begin{array}{c}
m 3(\{a\}) \\
m 3(\{b\}) \\
m 3(\{b, c\}) \\
m 3(\{c\}) \\
m 3(\{d\})
\end{array}\right]=\left[\begin{array}{c}
0.18 \\
0.15 \\
0.16 \\
0.33 \\
0.18
\end{array}\right]
$$

\section{(3) Computation of a Weight Value Using Bel and PI}

The values of Bel and Pl function can be computed by using bpa of $\mathrm{m} 3$.

$\operatorname{Bel}(\{a\})=m 3(\{a\})=0.18, \quad \operatorname{Bel}(\{b\})=m 3(\{b\})=0.15$,

$\operatorname{Bel}(\{c\})=m 3(\{c\})=0.33, \quad \operatorname{Bel}(\{d\})=m 3(\{d\})=0.18$

$\operatorname{Pl}(\{a\})=m 3(\{a\})=0.18, \quad \operatorname{Pl}(\{b\})=m 3(\{b\})+m 3(\{b, c\})=0.15+0.16=0.31$,

$\operatorname{Pl}(\{c\})=m 3(\{c\})+m 3(\{b, c\})=0.33+0.16=0.49, \quad \operatorname{Pl}(\{d\})=m 3(\{d\})=0.18$

The interval of focal elements using the values of Bel and $\mathrm{Pl}$ function is then

$$
\{a\}=[0.18,0.18],\{b\}=[0.15,0.31],\{c\}=[0.33,0.49],\{d\}=[0.18,0.18]
$$

In this case, the focal element $\{b\}$ ranges from 0.15 to 0.31 . This means that the belief interval of focal element $\{b\}$ is between 0.15 and 0.31 . Then the weight value of focal element is determined by averaging the values of belief interval. The final weight value is given below.

Weight value of $\{a\}=0.18$, Weight value of $\{b\}=0.23$, Weight value of $\{c\}=0.41$, Weight value of $\{d\}=0.18$ 
Table 3. Result after applying the combination rule

\begin{tabular}{c|c|c|c}
\hline Assessor & $\begin{array}{c}\text { Preference } \\
\text { relationship }\end{array}$ & $\begin{array}{c}\text { Focal } \\
\text { element }\end{array}$ & Bpa \\
\hline A & $\{a\}>\{b\}$ & $\{a\},\{b\}$ & $\{a\}=0.7,\{b\}=0.3$ \\
\hline B & $\{\mathrm{c}\}>\{\mathrm{d}\}$ & $\{\mathrm{c}\},\{\mathrm{d}\}$ & $\{\mathrm{c}\}=0.7,\{\mathrm{~d}\}=0.3$ \\
\hline C & $\{\mathrm{a}\}>\{\mathrm{c}\}$ & $\{\mathrm{a}\},\{\mathrm{c}\}$ & $\{\mathrm{a}\}=0.7,\{\mathrm{c}\}=0.3$ \\
\hline $\begin{array}{l}\text { Combination of A and B } \\
\text { (normal combination) }\end{array}$ & - & - & - \\
\hline $\begin{array}{l}\text { Combination of A and B } \\
\text { (improved combination) }\end{array}$ & - & $\mathrm{a}\},\{\mathrm{b}\},\{\mathrm{c}\},\{\mathrm{d}\}$ & $\begin{array}{c}\{\mathrm{a}\}=0.392,\{\mathrm{~b}\}=0.108, \\
\{\mathrm{c}\}=0.392,\{\mathrm{~d}\}=0.108\end{array}$ \\
\hline $\begin{array}{l}\text { Combination of A and C } \\
\text { (normal combination) }\end{array}$ & - & $\{\mathrm{a}\}$ & $\begin{array}{l}\{\mathrm{a}\}=0.49 \text { (before normalization) } \\
\{\mathrm{a}\}=1(\mathrm{after} \text { normalization) }\end{array}$ \\
\hline $\begin{array}{l}\text { Combination of A and C } \\
\text { (improved combination) }\end{array}$ & - & $\{\mathrm{a}\},\{\mathrm{b}\},\{\mathrm{c}\}$ & $\begin{array}{c}\{\mathrm{a}\}=0.784,\{\mathrm{~b}\}=0.108, \\
\{\mathrm{c}\}=0.108\end{array}$ \\
\hline
\end{tabular}

Table 4. Combination result of multi evaluation items

\begin{tabular}{l|l|l|l}
\hline \multicolumn{1}{c|}{ Assessor } & \multicolumn{1}{c|}{$\begin{array}{c}\text { Preference } \\
\text { relationship }\end{array}$} & \multicolumn{1}{c}{ Focal element } & \multicolumn{1}{c}{ Bpa } \\
\hline A & $\{a\}>\{b\},\{b\}>\{c\}$ & $\{a\},\{b\},\{c\}$ & $\{\mathbf{a}\}=\mathbf{0 . 5},\{b\}=0.3,\{c\}=0.2$ \\
\hline B & $\{a\}>\{b, c\}$ & $\{a\},\{b, c\}$ & $\{a\}=0.7,\{b, c\}=0.3$ \\
\hline $\begin{array}{l}\text { Combination of } \\
\text { A and B } \\
\text { (not exclusive } \\
\text { combination) }\end{array}$ & - & $\{a\},\{b\},\{c\},\{b, c\}$ & $\begin{array}{l}\{\mathbf{a}\}=\mathbf{0 . 7 0 1},\{b\}=0.152, \\
\{c\}=0.09,\{b, c\}=0.056\end{array}$ \\
\hline
\end{tabular}

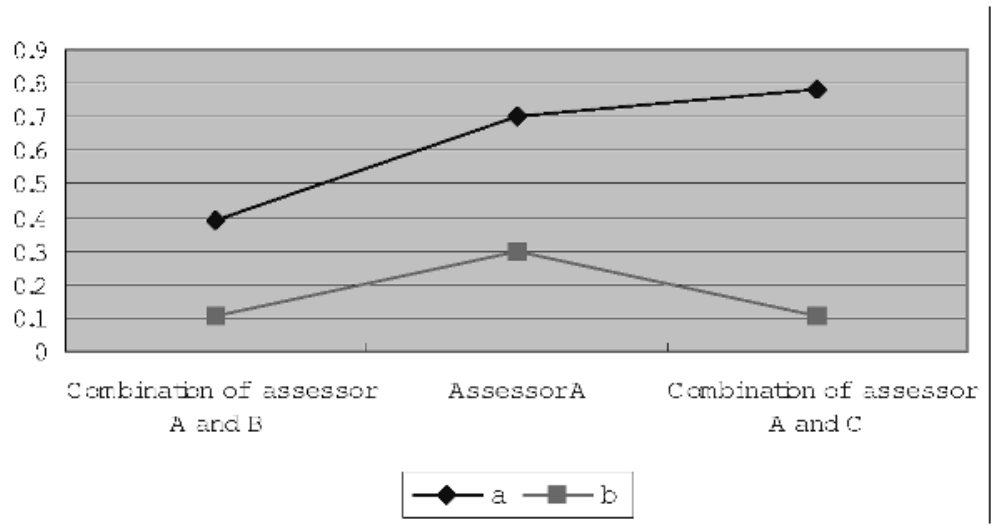

Fig. 1. Change of bpa on the focal element $\{a\}$ using the improved combination 


\section{Evaluation Result and Analysis}

\subsection{Comparison of the Combination Rule}

From the table 3, the results of normal combination of $\mathrm{A}$ and $\mathrm{B}$ are empty for any focal element because it is not possible to compute a weight value. However, the improved combination shows no empty set thanks to redistribution of empty set value.

\subsection{Analysis of Improved Combination Rule}

As shown in figure 1, the change of bpa on the focal element $\{a\}$ using the improved combination is reduced from 0.7 to 0.392 . This result indicates that assessor A assigns a high value to $\{\mathrm{a}\}$ but assessor B doesn't assigns any value to $\{\mathrm{a}\}$. The change of bpa on the improved combination for $\mathrm{A}$ and $\mathrm{C}$ in table 3 is raised from 0.7 to 0.784 . This result explains that both assessor $\mathrm{A}$ and assessor $\mathrm{B}$ assigns high value to $\{\mathrm{a}\}$.

Two combination results on bpa with focal element $\{b\}$ are same since the focal element $\{b\}$ is not common on the two combinations. On the other hand, bpa of focal element $\{a\}$ is 0.784 because focal element $\{a\}$ is common on the combination of both assessor $\mathrm{A}$ and $\mathrm{C}$. Table 4 illustrates that combination result of multi evaluation items.

The change of bpa on the combination A and B in table 4 is also raised from 0.5 to 0.701. Although assessor $\mathrm{B}$ had given preference relationship including multiple evaluation items such as $\{a\}>\{b, c\}$, we can obtain proper combination result.

\section{Conclusion}

The weight value calculation plays a key role in evaluation and selection of good quality software. This paper describes an quantitative method for calculating the weight value using DS theory. The proposed method eliminates the problem of assessor's subjective opinion and also improves the way of combining multiple assessors' opinion. The effectiveness of the new method has been verified with an example. The improved DS theory, however, still suffers from a great amount of numeric calculation $\left(\mathrm{O}\left(\mathrm{n}^{2}\right)\right)$ since it uses every exclusive bpas.

\section{References}

1. Software Quality Characteristics and Metrics - Part 1: Quality Characteristics \& SubCharacteristics, TTAS.IS-9126.1, October, 1998.

2. Evaluation of Sotfware Product - Part 5 : Process for Evaluators, TTAS.IS-14598.5, October, 1998.

3. Hae Sool Yang, Ha Yong Lee : Design and Implementation of Quality Evaluation Toolkit in Design Phase, Korea Information Science Society Journal (C), Vol. 3, No. 3, pp. 262274, June, 1997. 
4. Hae Sool Yang, Yong Gn Lee : Design and Implementation of Quality Evaluation supporting Tool for Software Specification, Information Science Society Journal (C), Vol. 3, No. 2, pp. 152-163, April, 1997.

5. Hae Sool Yang, Kee Hyun Kwon, Ha Yong Lee, Young Sik Jo, Yong Gn Lee, Jong Ho Park, Tae Gyoung Heo : Design and Implementation of Software Quality Evaluation Toolkit, Korea Information Processing Society Journal, Vol. 2, No. 2, pp. 185-198, 1995.

6. Catherine K. Murphy : Combining belief function when evidence conflicts, Decision Support System 29, pp 1-9, 2000.

7. L.A. Zadeh : Review of Mathematical theory of evidence, by G Shafer, AI Magazine5(3), pp 81-83, 1984.

8. Joseph Giarratano, Gray Riley, "Expert Systems", PWS Publishing Company, 1994.

9. Kum Sook We : Design of an Expert System for Software Quality Evaluation with Easy Weighting of Quality Element, Graduate School of Dongguk University Computer Engineering, Doctoral Dissertation, December, 1995.

10. Kum Sook We, Keum Suk Lee : Design of an Expert System for Software Quality Evaluation, Information Science Society Journal (B), Vol. 22, No. 10, pp. 1434-1444, October, 1995.

11. Yong Tae Do, Il Gon Kim, Jong Wan Kim, Chang Hyun Park : Artificial Intelligence Concept and Application, SciTech Press, pp. 77-96, 2001.

12. Hee Seung Kim : Artificial Intelligence and Application, Saengnung Press, pp. 199-216, 1994.

13. Jin Hyung Kim, Seung Su Park, En Ok Paek, Jung Yun Seo, Il Byong Lee : Artificial Intelligence Theory and Practice, SciTech Press, pp. 373-384, 1998.

14. Wong, S.K.M. and P.Lingras, "Representation of Qualitative User Preference by Quantitative Belief Functions", IEEE Transaction on Knowledge and Data Engineering, Vol.6, No.1, 1994, pp. 72-78.

15. Gye Sung Lee : An Efficient Dempster-Shafer Evidence Combination Scheme for Uncertainty Handling, Korea Information Processing Society Journal, Vol. 3, No. 4, pp. 908-914, July, 1996.

16. Gye Sung Lee : An Approximate Evidence Combination Scheme for Increased Efficiency, Korea Information Processing Society Journal(B), Vol.9-B, No. 1, pp. 17-22, February, 2002.

17. Jong Moo Lee, Ho Won Jung : An Application of Qualitative Preference to Software Quality Evaluation, Korea Operation Research and Management Science Society Journal, Vol. 25, No. 3, pp. 109-124, September, 2000. 